\title{
210 - Palliative Care in Dementia
}

\section{Continuous palliative sedation in nursing home residents with dementia suffering from extreme refractory neuropsychiatric symptoms}

Annelies E. Veldwijk-Rouwenhorst ${ }^{a, b}$, Martin Smalbrugge ${ }^{c}$, Sytse U. Zuidema ${ }^{\text {d }}$, Suzan A.J. Hanssen ${ }^{\text {a,b }}$, Raymond T.C.M. Koopmans ${ }^{\mathrm{a}, \mathrm{b}, \mathrm{e}}$, Debby L. Gerritsen ${ }^{\mathrm{a}, \mathrm{b}}$

${ }^{a}$ Radboud University Medical Center, Radboud Institute for Health Sciences, Department of Primary and Community Care, the Netherlands.

${ }^{\mathrm{b}}$ Radboudumc Alzheimer Center, Nijmegen, the Netherlands

${ }^{\mathrm{c}}$ Department of General Practice and Elderly Care Medicine, Amsterdam Public Health research institute, Amsterdam University Medical Centers Location VUmc, Amsterdam, the Netherlands

${ }^{d}$ Department of General Practice and Elderly Care Medicine, University of Groningen, University Medical Center Groningen, Groningen, the Netherlands

e De Waalboog "Joachim en Anna", Center for Specialized Geriatric Care, Nijmegen, the Netherlands

Introduction: Extreme neuropsychiatric symptoms (NPS) can be a heavy burden for nursing home (NH)residents, relatives and caregivers. When conventional treatments are ineffective or have intolerable side effects, extreme NPS can be considered refractory. In these situations, continuous palliative sedation (CPS) is sometimes administered. We explored the trajectory leading to CPS and its application in NH-residents with dementia and refractory NPS.

Methods: A qualitative interview study was performed in 2017. Relatives, elderly care physicians and other staff members involved with three NH-residents with dementia and extreme refractory NPS who received CPS were interviewed. These $\mathrm{NH}$-residents lived on dementia special care units of three $\mathrm{NHs}$ in the Netherlands. We used consecutive sampling to select participants. Medical files were studied. Semistructured interviews were conducted. Transcriptions were analyzed with thematic analysis, including directed content analysis.

Results: Nine in-depth interviews with fourteen participants were held. Analysis resulted in five main themes with several subthemes reflecting phases of the trajectory leading to CPS and the CPS application itself, a sixth main theme concerned evaluations thereof. According to the first theme (runup), the suffering of the $\mathrm{NH}$-resident was described as unbearable/an inner struggle. Participants still had hope for improvement. Concerning the second theme (turning point), hope was lost, participants were convinced they had tried everything and experienced feelings of powerlessness and failure. Regarding theme three (considering CPS), intermittent sedation was applied in all three cases and peer consultation was employed. Honoring the wish of the $\mathrm{NH}$-resident and therapeutic uncertainties, among others, were important subthemes. According to theme four (decision to start CPS), in each case one specific aspect was a decisive trigger for administering CPS. Concerning theme five (applying CPS) feelings of relief were experienced after starting with CPS.

Conclusions: The trajectory leading up to CPS in NH-residents with dementia and extreme refractory NPS was complex and burdensome, but the application led to relief and contentment of all those involved. We recommend to include external consultation in the decision process and to apply intermittent sedation as a preceding step when CPS is considered.

\section{Perceptions of Advance Care Planning of Community-dwelling People with Young-Onset Dementia} and their Family Caregivers: A Qualitative Interview Study 
Ton H.J. de Wit ${ }^{a}$, Jenny T. van der Steen ${ }^{a}$, Marjolein E. de Vugt ${ }^{b}$, Christian Bakker ${ }^{a}$, Raymond T.C.M. Koopmans ${ }^{\text {a.c }}$,

${ }^{a}$ Department of Primary and Community Care, Radboudumc Nijmegen, and Radboudumc Alzheimer Center, the Netherlands

${ }^{\mathrm{b}}$ Alzheimer Center Limburg, Maastricht, the Netherlands

${ }^{\mathrm{c} J o a c h i m}$ en Anna, center for specialized geriatric care, Nijmegen the Netherlands

Introduction: The impact of dementia before the age of 65 years differs from the impact on older persons and their family caregivers. Young-onset dementia (YOD) affects people in a more active phase of their life, in both private and working life, including the care for often young children. This often results in a different perspective of the future. Therefore, perceptions of advance care planning (ACP) may be distinct. The aim of this study is to better understand the experiences of people with YOD and their family caregivers with professional care and their views regarding quality of life, and how this relates to their perceptions regarding ACP.

Methods: For this qualitative interview study, as part of the Care4Youndem-study, semi-structured individual interviews were conducted with community-dwelling persons with YOD and their family caregivers in the Netherlands. It is expected that about 20 interviews will be needed to reach saturation. Interviews are coded with Atlas.ti and analyzed using inductive content analysis.

Results: Preliminary analyses of 8 interviews available so far indicate that persons with dementia hope that their life will continue as it is for as long as possible, with only minor problems and an acceptable quality of life. Individuals vary in the way to what they consider future care needs such as end of life decisions, advance directives or euthanasia. People with dementia have a tendency to postpone these discussions. Although possible admission to a long-term care facility is foreseen, persons with dementia mostly refer to their family caregiver to decide on their behalf.

Conclusion: By exploring issues and views regarding quality of life and ACP in both persons with YOD and their family caregivers, recommendations can be made to improve palliative care for people with YOD.

Increasing recognition of pre-death grief amongst carers of people living with dementia: Developing and evaluating an animation

Moore $\mathrm{KJ}^{1}$, Crawley $\mathrm{S}^{1}$, Vickerstaff $\mathrm{V}^{1}$, Cooper $\mathrm{C}^{2,3}$, Sampson $\mathrm{EL}^{1,4}$

${ }^{1}$ Marie Curie Palliative Care Research Department, UCL, London, UK

${ }^{2}$ Division of Psychiatry, UCL, London, UK

${ }^{3}$ Camden and Islington NHS Foundation Trust, London, UK

${ }^{4}$ Barnet, Enfield and Haringey Mental Health Trust, London, UK

Introduction: Family and friend carers may encounter pre-death grief while caring for someone with dementia. Grief theories suggest that acknowledging grief is important for processing difficult emotions, however, there is limited discourse in health and social care services to help carers recognise pre-death grief. We aimed to develop and evaluate a resource that could be used to increase awareness of carer pre-death grief in dementia.

Methods: We interviewed 150 UK carers and measured grief severity using the Marwit-Meuser Caregiver Grief Inventory. We asked carers whether they recognised they were experiencing grief and what they found helpful in managing grief. We reported our findings to a stakeholder panel including current and bereaved family carers and healthcare professionals working in dementia care. We designed 